J3eA, Journal sur l'enseignement des sciences et technologies de l'information et des systèmes, Volume 4, Hors-Série 2, 14 (2005)

DOI : http://dx.doi.org/10.1051/bib-j3ea:2005714

(C) EDP Sciences, 2005

\title{
Réalisation d'un banc d'essais thermique pour l'enseignement des systèmes non entiers
}

J. Sabatier, P. Melchior et A. Oustaloup

$$
\text { LAP }
$$

UMR 5131 CNRS - Équipe CRONE

Université Bordeaux 1 - ENSEIRB

351 cours de la Libération

F-33405 Talence CEDEX, France 


\title{
Réalisation d'un banc d'essais thermique pour l'enseignement des systèmes non entiers
}

\author{
Jocelyn SABATIER, Pierre MELCHIOR et Alain OUSTALOUP \\ LAP - UMR 5131 CNRS - Equipe CRONE \\ Université Bordeaux 1 - ENSEIRB \\ 351 cours de la Libération - F33405 TALENCE Cedex \\ Tél : +33(0)540006607 - Fax : +33(0)540006644 \\ Email : sabatier@lap.u-bordeaux1.fr - URL : http:॥www.lap.u-bordeaux1.fr
}

\begin{abstract}
Résumé : Cet article présente un banc d'essais pouvant être utilisé, à Bac +4 ou 5 , comme support pratique pour l'étude des systèmes non entiers, leur modélisation, simulation, identification, observation et commande. Ce banc d'essais est constitué d'un barreau d'aluminium entièrement isolé et chauffé à une extrémité. La grande longueur du barreau d'aluminium permet de le considérer comme un milieu semi-infini et de démontrer l'existence d'un transfert non entier liant le flux thermique appliqué à l'une de ses extrémités et la température à l'intérieur du barreau.
\end{abstract}

Mots clés : Automatique, Banc d'essais, Systèmes non entiers, Modélisation, Simulation, Identification, Observation, Commande

\section{1 - Introduction}

Ces dix dernières années ont vu l'émergence des systèmes à dérivées non entières (ou fractionnaires) [1][2][3][4] dans le domaine de l'Automatique et de la Robotique notamment au travers d'applications en :

- modélisation,

- identification,

- diagnostic,

- commande et commande robuste,

- planification de trajectoire.

Face à cet intérêt croissant, certaines universités ont même intégré l'étude des systèmes non entiers et de leurs applications dans leurs modules d'enseignement [5] (à l'image notamment de l'ENSEIRB).

Cet article s'inscrit dans cette évolution pédagogique puisqu'il présente un banc d'essais thermique pouvant être utilisé pour initier les étudiants à l'étude des systèmes non entiers. Outre une présentation générale des caractéristiques techniques de ce banc, cet article propose, afin d'illustrer ses possibilités en terme de pédagogie :

- la modélisation du banc par modèle non entier et la mise en évidence de l'intérêt de l'approche non entière pour caractériser les phénomènes de diffusion,

- son identification par modèle non entier,

- la simulation du modèle obtenu par différentes méthodes numériques avec comparaison à la réponse analytique de ce modèle et à la réponse temporelle du banc,

- la mise en œuvre d'un observateur d'état fractionnaire,

- la commande du banc par placement de pôles.

\section{2 - Description du banc d'essais}

Le banc d'essais reproduisant le comportement d'un système à dérivées non entières met en œuvre un système thermique de dimension semi-infinie, à savoir un barreau d'aluminium de grande longueur $(40 \mathrm{~cm})$ (figure 1). Comme l'illustre la figure 2, l'entrée de ce système est un flux thermique et sa sortie est la température du barreau mesurée à une distance $d$ de la surface chauffée.
Afin d'assurer un transfert de chaleur unidirectionnel, toute la surface du barreau est isolée (figure 3). Le flux thermique est produit par une résistance chauffante collée sur une extrémité du barreau (colle à haute conductivité thermique). Le flux maximal que peut produire la résistance est de $12 \mathrm{~W}$ (1A sous $12 \mathrm{~V})$.

Le flux thermique est contrôlé par un micro-ordinateur de type PC muni d'une carte entrée-sortie de type PCaxe (figure 4). L'interface de puissance séparant la carte entrée sortie et la résistance chauffante est un convertisseur PWM dont la tension d'entrée varie de 0 à $12 \mathrm{~V}$. Compte tenu de la fréquence de fonctionnement de ce convertisseur $(1 \mathrm{kHz})$ vis-à-vis de la dynamique du barreau, ses effets sont négligeables.

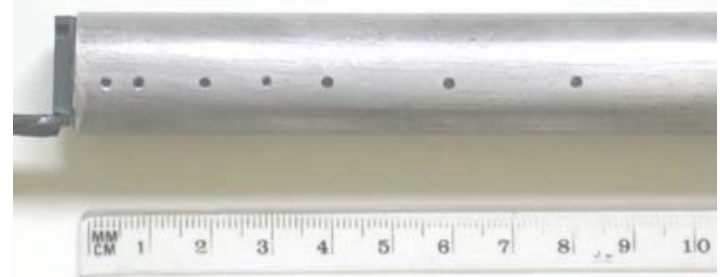

Figure 1 - Barreau d'aluminium, résistance chauffante 0 - $12 \mathrm{~W}$ (à gauche) et orifices de mesures

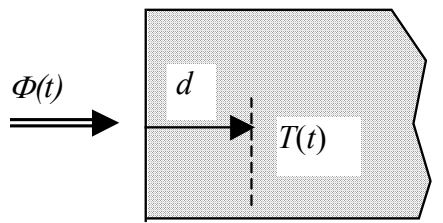

Figure 2 - Schéma du système thermique

La température du barreau est mesurée avec des sondes platines de type PT100 via un amplificateur de tension. Les sondes sont insérées à l'intérieur du barreau. Les orifices permettant le passage de ces sondes apparaissent sur la photographie de la figure 1. Ces orifices sont de faible diamètre $(2 \mathrm{~mm})$ afin de ne pas trop perturber le transfert thermique. Pour favoriser le transfert thermique vers les sondes thermiques, les orifices permettant la mesure sont 
garnis d'une pâte thermoconductrice avant le montage des sondes.

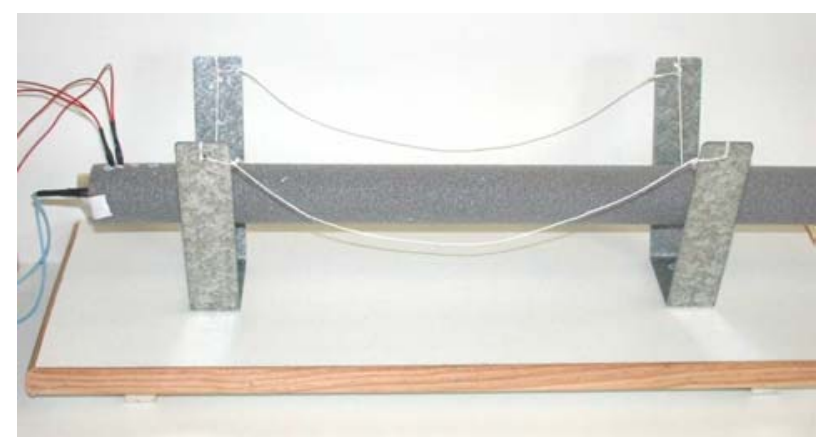

Figure 3 - Photographie du système thermique isolé, muni de la résistance chauffante et des sondes de température

L'amplificateur de tension délivre une information qui est convertie par la carte entrée-sortie au moyen d'un convertisseur analogique numérique 10 bits. L'erreur de quantification produite par la chaîne capteur / amplificateur / convertisseur est de $0.125{ }^{\circ} \mathrm{C}$.

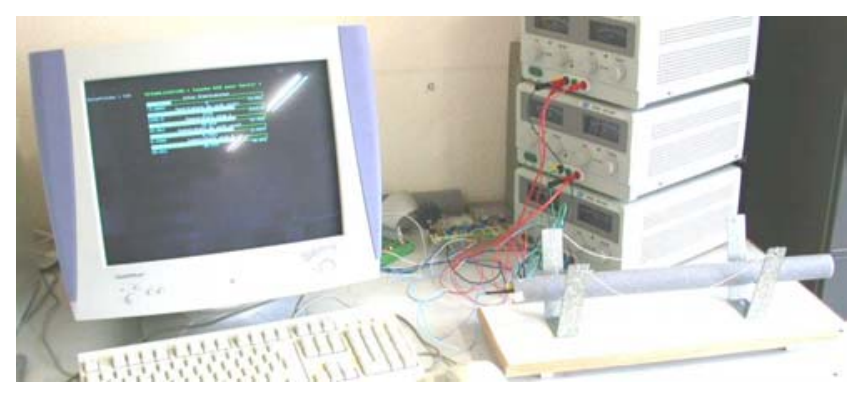

Figure 4 - Photographie du banc d'essais

\section{3 - Caractérisation du système thermique : approche analytique}

D’un point de vue pédagogique et théorique, cette partie est l'occasion de rappeler les différentes définitions des opérateurs de dérivation et d'intégration non entière [2, 13-14], mais aussi de faire apparaître les différences par rapport au cas entier, au niveau des propriétés de base liées à ces opérateurs.

D'une façon générale, l'intégrale d'ordre $\gamma$ d'une fonction $f(t)$ est définie par:

$$
{ }_{a} I_{t}^{\gamma} f(t)=\frac{1}{\Gamma(\gamma)} \int_{a}^{t}(t-\tau)^{\gamma-1} f(\tau) d \tau .
$$

$a=0$ correspond à la définition originelle de Riemann et $\mathrm{a}=-\infty$ correspond quant à elle à la définition originelle de Liouville. La dérivée d'ordre $\gamma$ d'une fonction $f(t)$ peut quant à elle être définie comme l'inverse à gauche de l'intégrale d'ordre $\gamma$. Pour $n-1<\gamma<n, n \in \mathbb{N}$ on a alors.:

$$
\begin{aligned}
{ }_{a} D_{t}^{\gamma} f(t) & =D_{a}^{n} I_{t}^{\gamma} f(t) \\
& =\frac{1}{\Gamma(n-\gamma)} \int_{a}^{t}(t-\tau)^{n-\gamma-1} D^{n} f(\tau) d \tau
\end{aligned} .
$$

Comme dans le cas entier, on montre facilement [19] que la transformée de Laplace de la dérivée non entière d'une fonction $f(t)$ est définie par :

$$
\begin{aligned}
\mathcal{L}\left\{{ }_{0} D_{t}^{\gamma} f(t)\right\} & =s^{\gamma} \mathcal{L}\{f(t)\}-\sum_{k=0}^{n-1} s^{k}\left[{ }_{0} D_{t}^{\gamma-k-1} f(t)\right]_{t=0} \\
& =s^{\gamma} \mathcal{L}\{f(t)\}-\sum_{k=0}^{n-1} c_{k} s^{k} .
\end{aligned}
$$

Du fait du manque de sens physique des constantes $c_{k}$, une définition différente de la dérivée non entière d'une fonction a été proposée par Caputo [14][19] :

$$
\begin{aligned}
{ }_{0}^{C} D_{t}^{\gamma} f(t) & ={ }_{0} I_{t}^{n-\gamma} D^{n} f(t) \\
& =\frac{1}{\Gamma(n-\gamma)} \int_{0}^{t}(t-\tau)^{n-\gamma-1} D^{n} f(\tau) d \tau .
\end{aligned}
$$

La transformée de Laplace de la dérivée non entière au sens de Caputo est alors donnée par :

$$
\mathcal{L}\left\{{ }_{0}^{C} D_{t}^{\gamma} f(t)\right\}=s^{\gamma} \mathcal{L}\{f(t)\}-\sum_{k=0}^{n-1} s^{\gamma-k-1}\left[{ }_{0} D_{t}^{k} f(t)\right]_{t=0},
$$

relation qui fait à présent intervenir des conditions initiales qui ont un sens physique.

Un exposé exhaustif des propriétés liées à ces définitions est présenté dans [13] et pourra être utilisé à des fins pédagogiques.

D'un point de vue pratique, l'étude du transfert de chaleur dans les milieux semi-infinis plans pour mettre en évidence que le système thermique qui compose le banc d'essais est un système non entier, est aussi l'occasion de mettre en évidence que phénomènes de diffusion et systèmes non entiers sont intimement liés.

Pour cela, le barreau est considéré comme étant un milieu semi-infini plan. Le milieu est considéré homogène, de conductivité $\lambda$, de diffusivité $\alpha$ et à température initiale nulle en tout point. Le milieu est soumis à une densité de flux $\varphi(t)$ sur la surface normale sortante $\vec{n}$. On considère en outre qu'il n'y a pas de perte au niveau de la surface d'application du flux.

Le transfert de chaleur $1 \mathrm{D}$ est régi par le système d'équations aux dérivées partielles [9] :

$$
\left\{\begin{array}{l}
\frac{\partial T(r, t)}{\partial t}=\frac{\alpha}{r^{p}} \frac{\partial}{\partial r}\left(r^{p} \frac{\partial T(r, t)}{\partial r}\right), 0<r<\infty, t>0 \\
-\lambda \frac{\partial T(r, t)}{\partial n}=\varphi(t), r=0, t>0 \\
T(r, t)=0,0 \leq r<\infty, t=0
\end{array}\right.
$$

où $p$ est une variable désignant le type de géométrie considérée.

L'étude d'un milieu semi-infini plan correspond au cas où $p=0$ et $r=x, x$ désignant l'abscisse du point de mesure de température à l'intérieur du milieu (figure 5). Le système (6) se simplifie et conduit alors à :

$$
\left\{\begin{array}{l}
\frac{\partial T(x, t)}{\partial t}=\alpha \frac{\partial^{2} T(x, t)}{\partial x^{2}}, 0<x<\infty, t>0 \\
-\lambda \frac{\partial T(x, t)}{\partial n}=\varphi(t), \quad x=0, t>0 \\
T(x, t)=0, \quad 0 \leq x<\infty, t=0
\end{array}\right.
$$




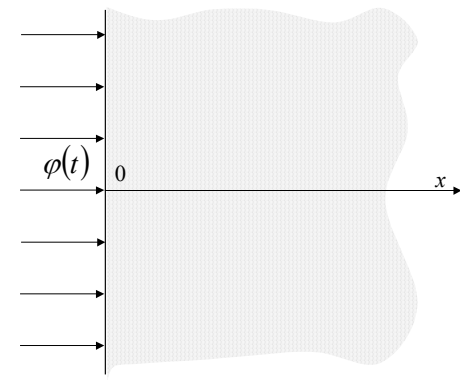

Figure 5 - Mur plan semi-infini

La transformation de Laplace de la première équation conduit à :

$$
\frac{\partial^{2} \bar{T}(x, s)}{\partial x^{2}}-\frac{s}{\alpha} \bar{T}(x, s)=0 \text { où } \bar{T}(x, s)=\mathcal{L}\{T(x, t)\},
$$

relation qui définit une équation différentielle par rapport à la variable $x$. La solution d'une telle équation est immédiate et s'exprime par :

$$
\bar{T}(x, s)=K_{1}(s) e^{-x \sqrt{s / \alpha}}+K_{2}(s) e^{x \sqrt{s / \alpha}} .
$$

La prise en compte des conditions aux limites conduit au transfert défini par :

$$
H(x, s)=\frac{\bar{T}(x, s)}{\bar{\varphi}(s)}=\frac{1}{\sqrt{s} \sqrt{\lambda \rho C_{p}}} \mathrm{e}^{-x \sqrt{s / \alpha}} .
$$

Deux cas d'études sont à distinguer.

Le premier correspond à l'étude de l'impédance thermique du milieu (soit $x=0$ ), c'est-à-dire du transfert défini par :

$$
H(0, s)=\frac{\bar{T}(0, s)}{\bar{\varphi}(s)} .
$$

Le second correspond à l'étude du transfert défini pour tout $x>0$ par :

$$
H(x, s)=\frac{\bar{T}(x, s)}{\bar{\varphi}(s)} .
$$

Dans le cas où $x=0$, le transfert (10) se simplifie en :

$$
H(0, s)=\frac{\bar{T}(0, s)}{\bar{\varphi}(s)}=\frac{1}{\sqrt{s} \sqrt{\lambda \rho C_{p}}},
$$

soit, dans le domaine temporel, les conditions initiales étant nulles :

$$
T(0, t)=\frac{1}{\sqrt{\lambda \rho C_{p}}}{ }_{0} I_{t}^{0.5} \varphi(t),
$$

relation traduisant un résultat maintenant bien connu : l'impédance thermique d'un milieu semi-infini plan est définie par un intégrateur d'ordre 0.5. Ce résultat, permettant d'exprimer analytiquement la température $T(0, t)$ uniquement en fonction de l'intégrale d'ordre $0.5 \mathrm{du}$ flux $\varphi(t)$, met ainsi en avant le caractère compact de l'opérateur de dérivation non entière. Il est vrai que la modélisation d'une telle impédance par une approche basée sur une méthode de discrétisation spatio-temporelle (méthode des éléments finis par exemple) conduit à une approximation par un modèle entier de très grande dimension.
Dans le cas où la mesure de température s'effectue à l'intérieur du milieu, soit $x>0$, le transfert $H(x, s)$ est donné par l'équation (10).

Dans le cadre d'un développement en série de $H(x, s)$, la fonction exponentielle s'exprime par :

$$
\mathrm{e}^{z}=\sum_{k=0}^{\infty} \frac{z^{k}}{k !} .
$$

Deux types de développement en série de $H(x, s)$ sont alors possibles.

Le premier développement, basé sur le développement direct de $\mathrm{e}^{-x \sqrt{s / \alpha}}$, conduit à :

$$
\begin{gathered}
\tilde{H}_{1, K}(x, s)=\frac{1}{\sqrt{\lambda \rho C_{p}}} \sum_{k=0}^{K} a_{k} s^{(k-1) / 2} \text { avec } \\
a_{k}=(-1)^{k} \frac{x^{k}}{\alpha^{k / 2} k !}
\end{gathered}
$$

soit, dans le domaine temporel :

$$
D^{0.5} T(x, t)=\frac{1}{\sqrt{\lambda \rho C_{p}}} \sum_{k=0}^{K} a_{k} D^{k / 2} \varphi(t) .
$$

Le second développement, inspiré de la méthode d'approximation de Padé d'un retard pur, soit :

$$
H(x, s)=\frac{1}{\sqrt{s} \sqrt{\lambda \rho C_{p}}} \mathrm{e}^{-x \sqrt{s / \alpha}}=\frac{1}{\sqrt{s} \sqrt{\lambda \rho C_{p}}} \frac{\mathrm{e}^{-\frac{x}{2} \sqrt{s / \alpha}}}{\mathrm{e}^{\frac{x}{2} \sqrt{s / \alpha}}}
$$

conduit à :

$$
\begin{gathered}
\tilde{H}_{2, K}(x, s)=\frac{1}{\sqrt{\lambda \rho C_{p}}} \frac{\sum_{k=0}^{K} a_{k}^{\prime} s^{k / 2}}{\sum_{k=0}^{K}\left|a_{k}^{\prime}\right| s^{(k+1) / 2}} \text { avec } \\
a_{k}^{\prime}=(-1)^{k} \frac{(x / 2)^{k}}{\alpha^{k / 2} k !},
\end{gathered}
$$

soit, dans le domaine temporel :

$$
\sum_{k=0}^{K}\left|a_{k}^{\prime}\right| D^{(k+1) / 2} T(x, t)=\frac{1}{\sqrt{\lambda \rho C_{p}}} \sum_{k=0}^{K} a_{k}^{\prime} D^{k / 2} \varphi(t)
$$

L'avantage du second développement réside dans deux propriétés fondamentales :

- $\widetilde{H}_{2, K}(x, s)$ est une fonction propre alors que $\widetilde{H}_{1, K}(x, s)$ ne l'est pas ;

- tendant vers zéro plus rapidement que $a_{k}$ (sa raison étant deux fois plus faible), la suite $a_{k}^{\prime}$ assure une convergence plus rapide de $\widetilde{H}_{2, K}(x, s)$ vers $H(x, s)$.

Comme pour le cas $x=0$, les transferts $\widetilde{H}_{1, K}(x, s)$ et $\widetilde{H}_{2, K}(x, s)$ s'expriment alors, dans le domaine temporel, par une équation différentielle non entière dont les ordres de dérivation sont multiples de 0.5. Par ailleurs, ces deux transmittances font apparaître une intégration pure d'ordre 0.5, qui rend impossible leur approximation par une 
transmittance entière de dimension finie sur tout l'espace des fréquences.

Cette analyse fait donc bien apparaître l'intérêt des systèmes à dérivées non entières pour la modélisation des phénomènes de diffusion. Le lien entre phénomènes de diffusion et systèmes non entier est également démontré dans les développements placés en annexe.

\section{4 - Caractérisation du système thermique : approche expérimentale par identification}

Le paragraphe précédent met en évidence que le système thermique qui compose le banc d'essais est un système non entier. Afin d'en obtenir un modèle de dimension réduite, son comportement est identifié au moyen d'un modèle non entier et en utilisant le module "Identification" du logiciel CRONE [6][7] et à partir de données temporelles expérimentales et non issues de la simulation des transmittances $\widetilde{H}_{1, K}(x, s)$ ou $\widetilde{H}_{2, K}(x, s)$.

D'un point de vue pédagogique et théorique, cette partie est l'occasion de faire découvrir aux étudiants les diverses méthodes qui ont été développées pour identifier des systèmes non entiers, à savoir :

- méthode à erreur d'équation avec estimation paramétrique par discrétisation de l'équation différentielle [20],

- méthode à erreur d'équation avec estimation paramétrique par la méthode des filtres des variables d'état non entiers [21],

- méthode à erreur de sortie avec structure de modèle basé sur sa décomposition en élément simple [22] ou son approximation fréquentielle [17], ou bien sa décomposition sur une base de Laguerre étendue [23].

D'un point de vue pratique, cette partie est l'occasion d'étudier la mise en œuvre d'une des méthodes précédemment citées. La méthode utilisée ici est une méthodes à erreur de sortie, les ordres de dérivation étant estimés au même titre que les coefficients. Cette méthode exploite des techniques d'optimisation non linéaires tel que l'algorithme de Marquart pour la minimisation d'un critère basé sur l'erreur de sortie du modèle.

Pour ce faire et comme l'illustre la figure 6 , le système thermique est sollicité par une séquence binaire pseudo aléatoire (sbpa). La réponse en température du système thermique est présentée par la figure 6. La température est mesurée à une distance $d=5 \mathrm{~mm}$ de la surface chauffée, mais le même travail pourrait être effectué en considérant des distances différentes à partir des différents trous percés dans le barreau. Il faut cependant veiller à ne pas se rapprocher trop de l'extrémité non chauffée pour que l'hypothèse d'un milieu semi-infini soit valide.

La mise en œuvre du module identification du logiciel CRONE [6][7] permet alors d'obtenir le modèle non entier suivant :

$$
H(s)=\frac{T(s)}{\varphi(s)}=\frac{-0.078121 s^{0.5}+0.12710}{3.0641 s^{1.5}+0.030475 s+s^{0.5}} .
$$

Le numérateur de la transmittance $H(s)$ a un zéro à partie réelle positive $z_{1}=2.6495$ qui permet de conclure que le modèle est à non minimum de phase. Les valeurs propres de cette transmittance sont $\lambda_{d 1}=-5 \times 10^{-3}+\mathrm{j} 5.7 \times 10^{-2}$, $\lambda_{d 2}=-5 \times 10^{-3}-\mathrm{j} 5.7 \times 10^{-2}, \lambda_{d 3}=0$. Ces valeurs propres ne génèrent pas de pôles, la notion de pôles et de valeurs propres étant différentes dans le cas de systèmes non entiers.

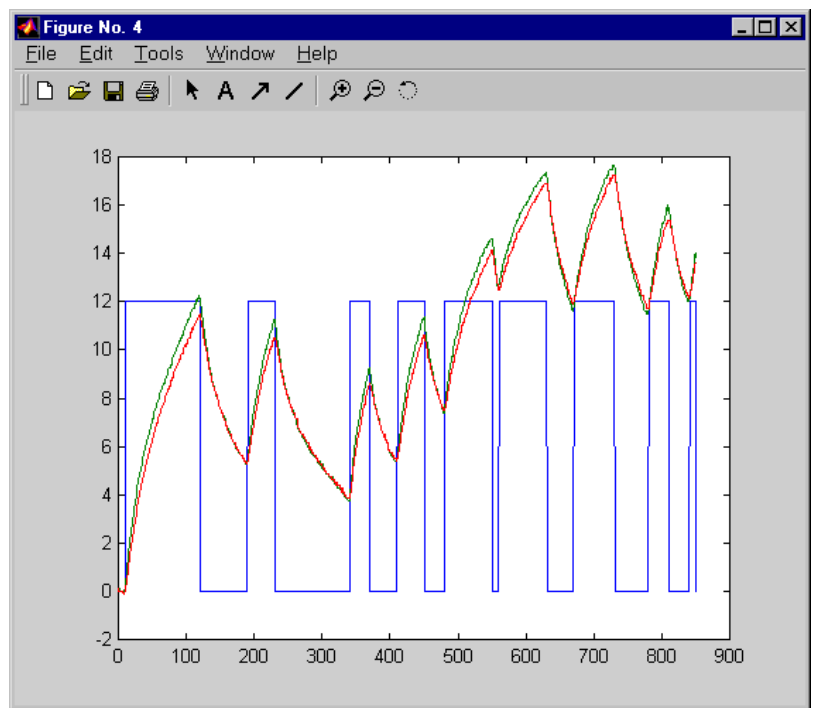

Figure 6 - Identification du banc d'essais : représentation du signal sbpa (créneaux), et comparaison des réponses du système thermique au signal sbpa et du modèle identifié

\section{5 - Simulation temporelle des modèles obtenus}

D’un point de vue pédagogique et théorique, l'étude de différentes méthodes de simulation peut être l'occasion de définir la réponse analytique d'un système non entier mais également de définir les différentes approximations de l'opérateur d'intégration non entière.

$\mathrm{Si}$ on considère des systèmes décrits par l'équation différentielle non entière

$$
\mathcal{S}: \sum_{k=0}^{N_{a}} a_{l}\left(\frac{d}{d t}\right)^{\gamma_{a_{k}}} y(t)=\sum_{k=0}^{N_{b}} b_{m}\left(\frac{d}{d t}\right)^{\gamma_{b_{k}}} u(t),
$$

dans laquelle $a_{N_{a}}=1$, les ordres $\gamma_{a_{k}}$ et $\gamma_{b_{k}}$ vérifiant les relations :

$$
\gamma_{a_{k}}=\frac{k}{q} \quad \text { et } \quad \gamma_{b_{k}}=\frac{k}{q}, \quad \text { avec } \quad q \in \mathbb{N},
$$

les ordres de dérivation $\gamma_{a k}$ et $\gamma_{b k}$ sont dits commensurables [12] (ils sont tous multiples d'un même rationnel $1 / q$ ),

Le système (22) est supposé linéaire, causal et relaxé à $t=0$. Sous ces hypothèses, le passage de l'équation (22) à la fonction de transfert est possible, soit [11]:

$$
\frac{Y(s)}{U(s)}=\frac{B(s)}{A(s)}=\sum_{k=0}^{N_{B}} b_{k} s^{\frac{k}{q}} / \sum_{k=0}^{N_{A}} a_{k} s^{\frac{k}{q}} .
$$

Le calcul de l'expression analytique de la sortie $y(t)$ de (22) figure dans [10-12]. Celle-ci est fondamentalement basée sur la décomposition modale d'un système non entier obtenue par décomposition en élément simple de la transmittance (24). La sortie $y(t)$ de (22) s'exprime ainsi par une combinaison linéaire d'éléments, appelés modes propres, définis par $H(s)=\mathcal{L}^{-1}\left\{\left(1 /\left(s^{\gamma}-\lambda_{l}\right)\right) q^{q_{i}}\right\} * \mathbf{u}(t)$ où $q_{i}$ est un nombre entier reflétant la multiplicité du $l^{\text {ième }}$ mode de valeur $\quad \lambda_{l}=\left|\lambda_{l}\right| e^{i \varphi_{l}} \quad$ (solution de l'équation $\left.\sum_{k=0}^{N_{A}} a_{k} s^{k}=0\right)$. 
Lorsque la valeur $\lambda_{l}$ est non nulle, la transformation inverse de Laplace de $H(s)$ est donnée par [11] :

$$
h(t)=h_{\exp }(t)+\sum_{k=0}^{q_{i}-1} h_{\text {ap }}(k, t),
$$

où

$$
h_{\exp }(t)=\sum_{k=1}^{\text {nbre pôles }} \frac{p_{k}}{\lambda_{l} q_{i}} Q_{q_{i}-1}\left(\frac{1}{\gamma}, t p_{k}\right) \mathbf{e}^{t p_{k}},
$$

et

$$
h_{a p}(k, t)=\frac{1}{\pi} \int_{0}^{\infty} \frac{e^{-t x}(-1)^{k}\left(\begin{array}{l}
q_{i} \\
k
\end{array}\right)\left(\lambda_{l}\right)^{k} x^{\gamma\left(q_{i}-k\right)} \sin \left[\gamma \pi\left(q_{i}-k\right)\right]}{\left[x^{2 \gamma}-2 \lambda_{l} x^{\gamma} \cos (\gamma \pi)+\lambda_{l}^{2}\right]^{q_{i}}} d x,
$$

les pôles $p_{k}$ du système étant définis par :

$$
p_{k}=\left|p_{k}\right| \mathrm{e}^{j \theta_{k}}, \operatorname{avec}\left\{\begin{array}{l}
\left|p_{k}\right|=\left(\left|\lambda_{l}\right|\right)^{1 / \gamma} \\
\theta_{k}=\frac{\arg \left(\lambda_{l}\right)}{\lambda}+\frac{2 k \pi}{\gamma} \\
-\frac{\gamma}{2}-\frac{\arg \left(\lambda_{l}\right)}{2 \pi}<k<\frac{\gamma}{2}-\frac{\arg \left(\lambda_{l}\right)}{2 \pi}
\end{array},\right.
$$

et où $Q_{k}(x, y)$ est un polynôme à deux variables défini par :

$$
\left\{\begin{array}{l}
Q_{0}(x, y)=x \\
\kappa Q_{\kappa}(x, y)=(x y+x-y) Q_{\kappa-1}(x, y)+x y \frac{\partial}{\partial y} Q_{\kappa-1}(x, y)
\end{array}\right.
$$

L'équation (25) met en évidence la décomposition structurelle de $h(t)$ en deux parties :

- la première, appelée mode exponentiel, résultant du calcul des résidus en chaque pôle ;

- la seconde, appelée multimode apériodique, résultant du calcul d'une intégrale le long de la coupure du plan complexe et caractérisée par une dynamique apériodique à mémoire longue.

Fort de cette analyse et d'un point de vue pratique, il est à présent possible de procéder à la comparaison de différentes méthodes de simulation. La plupart de ces méthodes sont basées sur l'approximation dans le domaine fréquentiel ou temporel de l'opérateur de dérivation non entière $s^{\gamma}$.

- Dans le domaine fréquentiel, on cherche comme l'illustre le schéma de la figure 7, à approximer l'opérateur de dérivation non entière $s^{\gamma}$ par une transmittance dont les pôles et les zéros sont liés par les relations de récurrence [3], à savoir :

$$
s^{\gamma} \approx D_{N}(s)=K \prod_{i=1}^{N}\left(1+\frac{s}{\omega_{i}^{\prime}}\right) / \prod_{i=1}^{N}\left(1+\frac{s}{\omega_{i}}\right)
$$

avec

$$
\begin{aligned}
& \alpha \eta=10 \frac{\log \left(\frac{\omega_{h}}{\omega_{b}}\right)}{N} \quad \alpha=10^{\gamma \log (\alpha \eta)} \\
& \omega_{1}=\omega_{b} \sqrt{\eta} \quad \omega_{1}^{\prime}=\alpha \omega_{1} \quad \omega_{i+1}=\alpha \eta \omega_{i} \\
& \omega_{i+1}^{\prime}=\alpha \eta \omega_{i}^{\prime}
\end{aligned}
$$

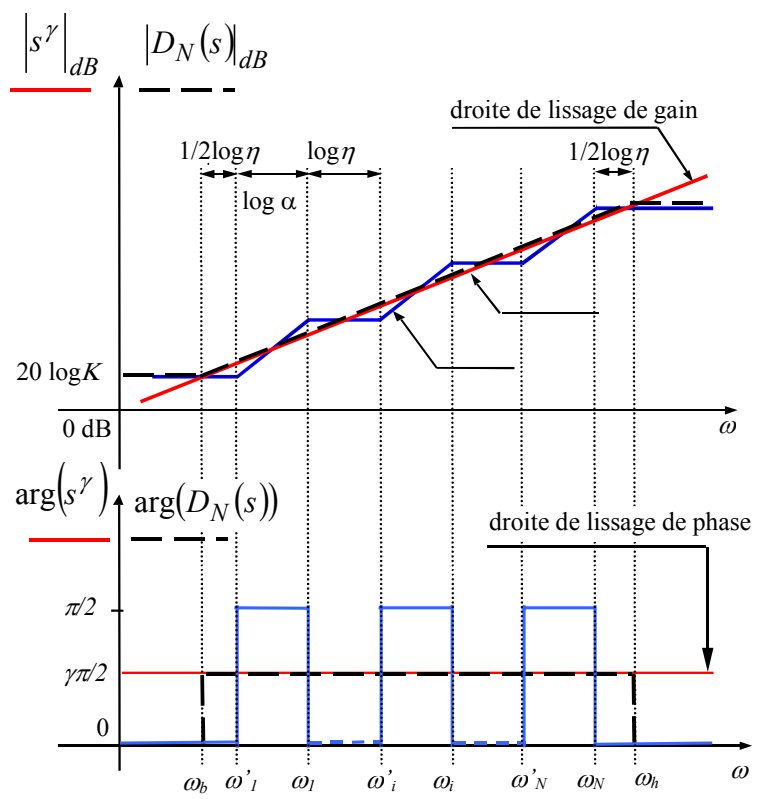

Figure 7 - Synthèse de l'opérateur $s^{\gamma}$ au moyen d'une distribution récursive de pôles et de zéros

Une amélioration de cette méthode est proposée dans [17].

- Dans le domaine temporel, l'approximation la plus répandue est celle issue de la définition de Grünwald, soit [1]

$$
D^{\gamma} f(K h) \approx \frac{1}{h^{\gamma}} \sum_{k=0}^{K}(-1)^{k}\left(\begin{array}{l}
\gamma \\
k
\end{array}\right) f((K-k) h),
$$

où $h$ désigne la période d'échantillonnage. Cette approximation résulte de l'utilisation de la formule d'Euler ( $q$ désignant l'opérateur d'avance) :

$$
s^{\gamma} \rightarrow\left(\frac{1}{h}\right)^{\gamma}\left(1-q^{-1}\right)^{\gamma}
$$

et du développement de cette formule. Cependant d'autres approximations peuvent être utilisées pour améliorer la précision des calculs [15] [16] :

$$
\begin{gathered}
s^{\gamma} \rightarrow\left(\frac{2}{h}\right)^{\gamma}\left(\frac{1-q^{-1}}{1+q^{-1}}\right)^{\gamma} \text { approximation de Tustin } \\
s^{\gamma} \rightarrow\left(\frac{8}{7 h}\right)^{\gamma}\left(\frac{1-q^{-1}}{1+q^{-1} / 7}\right)^{\gamma} \text { approximation d'Al-Alaoui. }
\end{gathered}
$$

Par rapport au banc d'essai proposé, on peut alors imaginer comparer ces méthodes de simulation sur le transfert de chaleur caractérisé par la transmittance (13) dont l'expression analytique de la réponse indicielle est donnée par :

$$
h(t)=\frac{1}{\sqrt{\lambda \rho C_{p}}} \frac{1}{\Gamma(3 / 2)} t^{1 / 2} .
$$

Pour chacune des méthodes testées, on pourra notamment comparer la complexité de mise en œuvre et la précision obtenue compte tenu de la nécessité de tronquer la dimension infinie des relations de récurrence donnant $h(t)$. 


\section{6 - Observation de l'état du système non entier}

D’un point de vue pédagogique et théorique, cette partie est l'occasion de définir la notion de représentation d'état pour les systèmes non entiers et de mettre en évidence la différence entre les notions de valeurs propres de la matrice d'état, de modes et de pôles.

D'après la condition de commensurabilité, le passage de la relation (22) à une représentation d'état non entière est possible. Elle est de la forme

$$
\left\{\begin{array}{l}
D^{(\gamma)} \mathbf{x}(t)=\mathbf{A} \mathbf{x}(t)+\mathbf{B} \mathbf{u}(t) \\
\mathbf{y}(t)=\mathbf{C} \mathbf{x}(t)+\mathbf{E} \mathbf{u}(t)
\end{array} .\right.
$$

Une autre représentation appelée représentation diffusive [18] est également possible pour un système non entier caractérisé par une transmittance de la forme (qui peut être obtenue par décomposition en élément simple) :

$$
H(s)=\sum_{k=1}^{N} \frac{\delta_{k}}{\left(p^{\gamma_{k}}+a_{k}\right)^{n_{k}}} \quad n_{k} \in \mathbb{N},
$$

Cependant l'obtention d'une telle représentation est subordonnée au calcul des pôles du système (voir en annexe).

Des résultats sur l'observabilité des systèmes non entiers sont disponibles dans [24] et [25].

D'un point de vue pratique, le banc d'essai proposé permet la mise en œuvre d'observateurs de Lunberger non entiers. Connaissant le flux de chaleur $\varphi(t)$ appliqué au barreau et connaissant la température $\theta_{\mathrm{s}}(\mathrm{t})$ en un point du barreau, on souhaite par exemple estimer la température $\theta_{0}(\mathrm{t})$ en un autre point de la barreau supposé inaccessible (par exemple la surface chauffée). A partir des développements du paragraphe 1, le système thermique peut se caractériser par le schéma de la figure 8, au moyen de deux fonctions de transfert fractionnaires $G_{0}(p)$ et $G_{\mathrm{r}}(p)$ telles que :

$$
\theta_{0}(t)=\mathcal{L}^{-1}\left[G_{0}(s)\right] * \varphi(t) \quad \theta_{r}(t)=\mathcal{L}^{-1}\left[G_{r}(p)\right] * \theta_{0}(t) .
$$

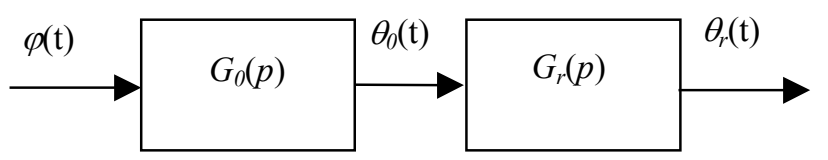

Figure 8 - Modélisation du procédé thermique en vue de l'observation de la température $\theta_{0}(t)$

En utilisant les formes compagnes d'observabilité liées aux transmittances $G_{0}(p)$ et $G_{\mathrm{r}}(p)$, le système thermique admet une représentation d'état analogue à la représentation donnée par la relation (35) avec :

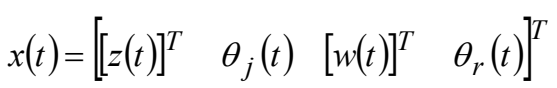

$[z(t)]=\left[\begin{array}{lll}z_{1}(t) & \cdots & z_{m}(t)\end{array}\right]^{T} \quad[w(t)]=\left[\begin{array}{llll}w_{1}(t) & \cdots & w_{n}(t)\end{array}\right]^{T}$.

Un observateur de Luenberger pour ce système est par conséquent de la forme :

$$
\left\{\begin{array}{l}
D^{\gamma} \hat{\mathbf{x}}(t)=\mathbf{A} \hat{\mathbf{x}}(t)+\mathbf{B} \varphi(t)+\mathbf{K}\left(\theta_{r}(t)-\hat{\theta}_{r}(t)\right) \\
\hat{\theta}_{r}(t)=\mathbf{C} \hat{\mathbf{x}}(t)+\mathbf{E} \varphi(t)
\end{array}\right.
$$

où

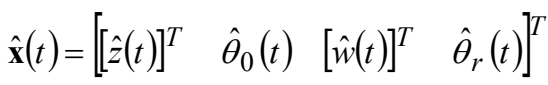

Les méthodes de discrétisation décrites au paragraphe 5 pourront être utilisées pour implanter un tel observateur.

\section{7 - Commande par placement modal}

L'objectif est à présent de réguler la température $T(s) \mathrm{du}$ barreau, mesurée à $5 \mathrm{~mm}$ de la surface chauffée.

D'un point de vue pédagogique et théorique, cette partie est également l'occasion d'étudier l'influence de la position des pôles ou des valeurs propres du système non entier sur sa stabilité et sur son degré de stabilité. On pourra pour cela s'attacher à étudier la réponse d'un système de la forme :

$$
\mathcal{S}_{1}: H_{1}(p)=\frac{1}{p^{\gamma}+1}, \quad \text { où } \quad 0<\gamma<2,
$$

désigné par système du premier ordre non entier, et d'un système de la forme :

$$
\begin{gathered}
\mathcal{S}_{2}: H_{2}(p)=\frac{1}{p^{2 \gamma}+2 p^{\gamma} \cos (\theta)+1}, \\
0<\gamma<2 \quad \text { et } \quad 0<\theta<\pi-\gamma \pi / 2,
\end{gathered}
$$

où

désigné par système du second ordre non entier.

Notons que tout système non entier défini par (22) peut se décomposer sur la base de sous systèmes de types $\mathcal{S}_{1}$ et $\mathcal{S}_{2}$ dans lesquels on a effectué un changement de variable, $\mathcal{S}_{1}$ correspondant à un mode propre réel, $\mathcal{S}_{2}$ étant déterminé à partir de la somme de 2 modes propres complexes conjugués.

La figure 9 présente la réponse du système $\mathcal{S}_{1}$, défini par la relation (40), pour plusieurs valeurs de $\gamma$. Cette figure permet de définir 3 classes de comportement :

- un comportement hyper amorti pour $0<\gamma<1$ (le système est dit hyper amorti) ;

- un comportement sous amorti pour $1<\gamma<2$ (le système est dit sous amorti);

- un comportement avec amortissement critique pour $\gamma=1$.

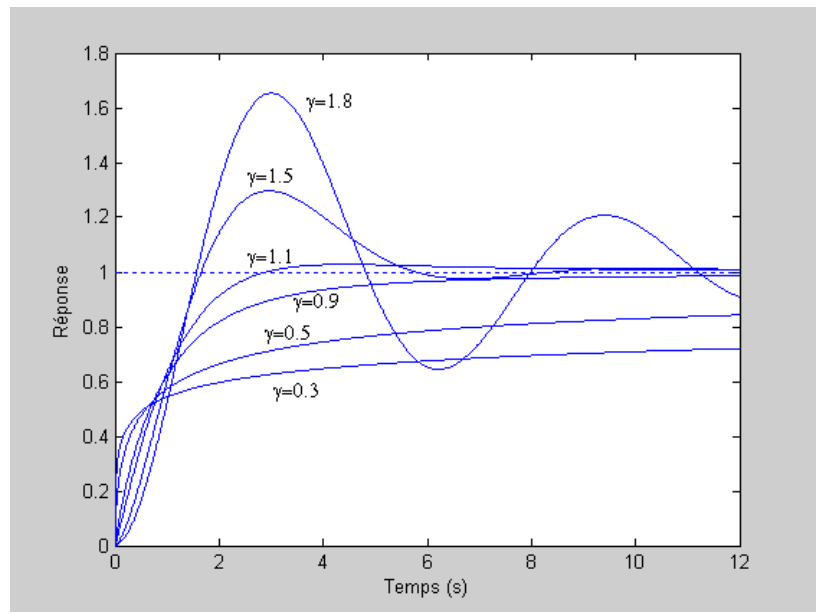

Figure 9 - Réponse du système $\mathcal{S}_{1}$ pour plusieurs valeurs de $\gamma$ 
Pour un système du second ordre non entier, il existe plusieurs couples de paramètres $\{\gamma, \theta\}$ qui conduisent à des caractéristiques temporelles voisines. Il est ainsi possible de définir des courbes d'iso-premier dépassement réduit pour les couples $\{\gamma, \theta\}$ qui conduisent à des comportements sous amortis (figure 10) et des courbes d'iso-temps de réponse à $95 \%$ pour les couples $\{\gamma, \theta\}$ qui conduisent à des comportements hyper amortis (figure 11).

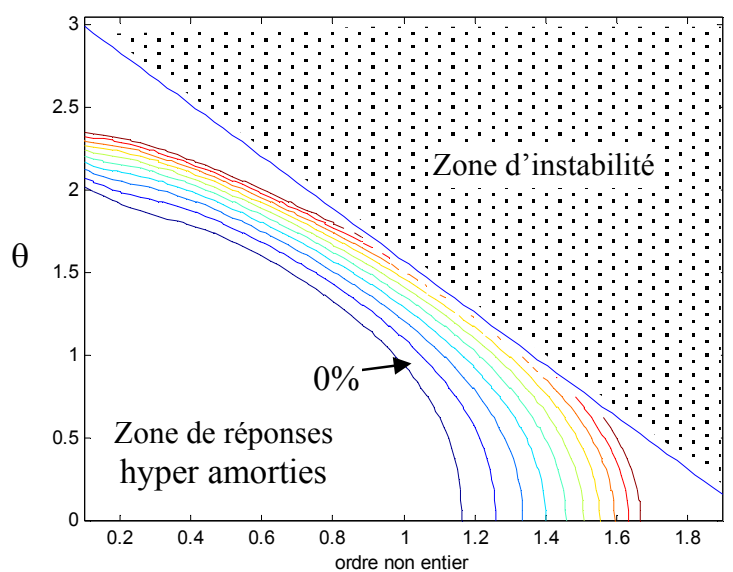

Figure 10 - Courbes d'iso-dépassement réduit de niveau 0\%, $10 \%, 20 \%, 30 \%, 40 \%, 50 \%, 60 \%, 70 \%, 80 \%, 90 \%$, 100\% (la plus proche de la zone d'instabilité) pour un système de type $\mathcal{S}_{2}$

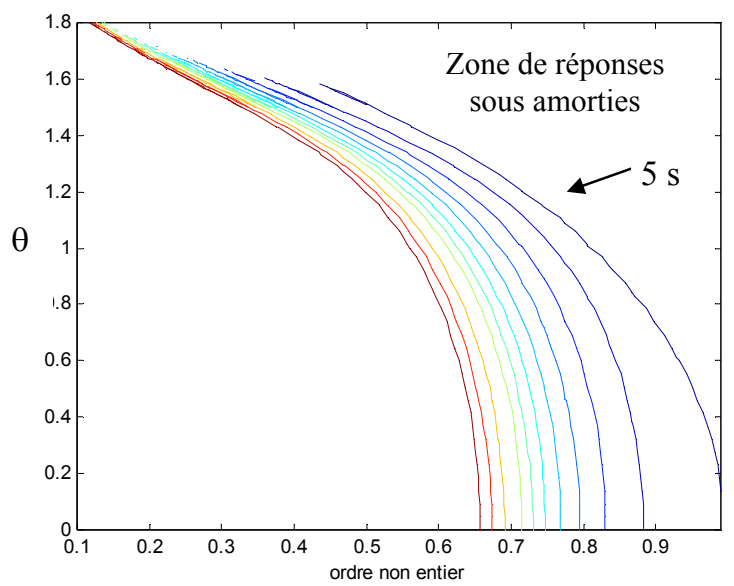

Figure 11 - Courbes d'iso-temps de réponse à 95\% de niveau $5 \mathrm{~s}$, $10 s, 15 s, 20 s, 25 s, 30 s, 35 s, 40 s, 50 s, 60 s, 70 s$ pour un système de type $\mathcal{S}_{2}$

En utilisant ces courbes, le temps de réponse à $95 \%$ de tout système d'ordre non entier de la forme

$$
\mathcal{S}: H(p)=\frac{1}{\frac{p^{2 \gamma}}{a^{2}}+\frac{2 p^{\gamma} \cos (\theta)}{a}+1}, 0<\gamma<2, \quad a \in \mathbb{R},
$$

peut être défini à partir de la figure 11 et en utilisant la relation :

$$
t_{95 \%}(\mathcal{S})=\frac{t_{95 \%}\left(\mathcal{S}_{2}\right)}{a^{1 / n}}
$$

Il va s'en dire que ces réseaux de courbes fournissent des renseignements importants pour effectuer le choix des pôles en boucle fermée dans le cadre d'une approche de type placement de pôles. On notera que la zone d'instabilité apparaissant sur la figure 10 peut être déterminé à partir du théorème de stabilité qui suit.
Théorème [12]. Le système non entier caractérisé par la transmittance

$$
H(s)=\frac{1}{\left(s^{\gamma}-\lambda\right)^{n}} \quad n \in \mathbb{N}, \quad \lambda \in \mathbb{C}
$$

est stable si et seulement si $|\arg (\lambda)| \leq \gamma \frac{\pi}{2}$.

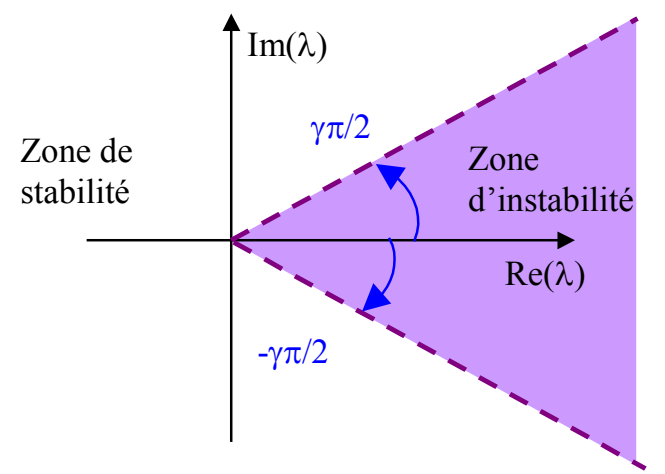

Figure 12 - Définition des zones du plan complexe assurant la stabilité du système caractérisé par la transmittance $H(s)$

D'un point de vue pratique la structure de commande de la figure 13 pourra être utilisée pour réaliser la commande du banc où les polynômes $R(s)$ et $S(s)$ et la transmittance $F(s)$ sont donnés par :

$$
\begin{aligned}
& R(s)=\sum_{k=0}^{N_{R}} r_{k} s^{\frac{k}{q}} \quad S(s)=\sum_{k=0}^{N_{S}} s_{k} s^{\frac{k}{q}} \\
& F(s)=\sum_{k=0}^{N_{F}} f_{k} s^{\frac{k}{q}} / \sum_{k=0}^{N_{E}} e_{l} s^{\frac{k}{q}}, \quad q=2 .
\end{aligned}
$$

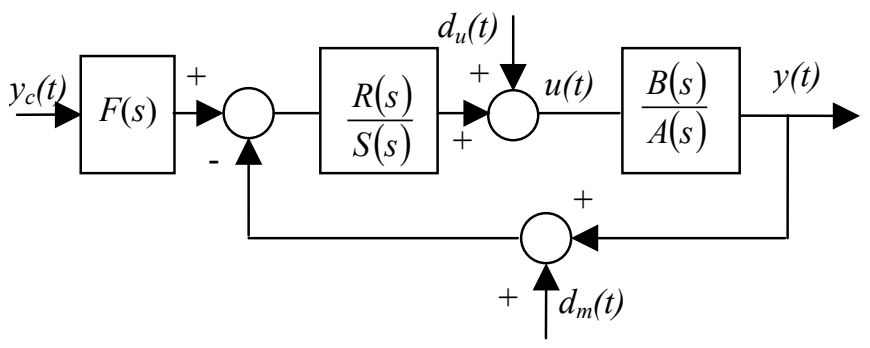

Figure 13 - Structure de commande utilisée

Le polynôme caractéristique du système de la figure 13 est défini par :

$$
A_{b f}(s)=S(s) A(s)+R(s) B(s) .
$$

La commande par placement modal consiste alors à choisir le polynôme non entier $A_{b f}(s)$ et à calculer les polynômes $R(s)$ et $S(s)$ pour que la relation (48) soit satisfaite. Le polynôme $A_{b f}(s)$ est choisi de sorte que l'ensemble des spécifications en rejet de perturbations soient satisfaites. La transmittance $F(s)$ est par la suite synthétisée pour tenir compte des spécifications en suivi de trajectoire.

Comme dans le cas des systèmes à dérivées entières, la relation (48) est une équation de Bezout, que l'on résout après avoir posé une série de contraintes sur les degrés des polynômes $R(s)$ et $S(s)$. Une description détaillée de cette méthode de commande dans le cas de systèmes non entiers est présentée dans [8]. 
Dans le cas du banc d'essais précédemment décrit, le polynôme $A_{b f}(s)$ est choisi égal à (voir également [8] pour des commentaires sur le choix de ce polynôme) :

$$
A_{b f}(s)=\left(\frac{s^{2}}{\omega_{0}^{2}}+\frac{2 \zeta}{\omega_{0}} s+1\right)\left(\frac{s^{0.5}}{\omega_{0}^{0.5}}+1\right)^{3}\left(\frac{s^{0.5}}{10^{0.5} \omega_{0}^{0.5}}+1\right)^{2} \text {. }
$$

avec $\omega_{0}=0.5$ et $\zeta=0.8$, ce qui conduit à :

$$
R(s)=7.8641+45.229 s^{0.5}+131.55 s+131.18 s^{1.5}+268.51 s^{2}
$$
et

$S(s)=19.078 s+15.236 s^{1.5}+12.107 s^{2}+4.8617 s^{2.5}+0.73846 s^{3}$.

Suite à son approximation par un transmittance d'ordre entier, l'implantation de ce régulateur est effectuée en utilisant l'approximation d'Euler $s=\left(1-z^{-1}\right) / T_{e}$, avec pour période d'échantillonnage $T_{e}=50 \mathrm{~ms}$.

La figure 14 présente la réponse du système en boucle fermée à un échelon de consigne de $2^{\circ} \mathrm{C}$ depuis une température initiale de $34^{\circ} \mathrm{C}$. La cassure apparaissant à l'instant $15 \mathrm{~s}$ est due à la saturation de la commande lors des premiers instants de la réponse.

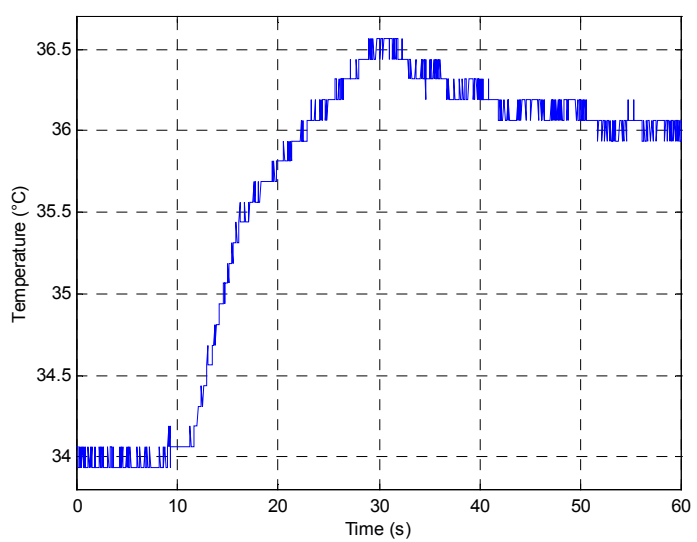

Figure 14 - Réponse du système en boucle fermée à un échelon de consigne de 2 degrés

\section{8 - Conclusion}

Cet article présente un banc d'essais thermique dont la conception s'est voulue la plus flexible possible pour permettre l'initiation à de nombreuses applications des systèmes à dérivées non entières. A titre d'illustration, l'article montre l'utilisation qui peut en être faite pour la modélisation, la simulation, l'identification, l'observation et la commande de systèmes non entiers. Mais on peut également imaginer se servir de ce banc pour bien d'autres applications, à savoir, toujours dans le cadre de systèmes non entiers :

- l'étude de méthodes de planification de trajectoire,

- le diagnostic et la détection de défauts (détection d'usure si le barreau d'aluminium est remplacé par un outil d'usinage, diagnostic thermique si le barreau d'aluminium est remplacé par un composant électronique et son radiateur).

\section{Annexe}

Cette annexe donne une représentation d'état différente de la représentation (35), appelée représentation diffusive pour un système non entier caractérisé par une transmittance de la forme (qui peut être obtenue par décomposition en éléments simples) :

$$
H(s)=\sum_{k=1}^{N} \frac{\delta_{k}}{\left(p^{\alpha_{k}}+a_{k}\right)^{n_{k}}} .
$$

Pour simplifier la présentation on posera ici $\delta_{k}=1$ et $n_{k}=1$ et $N=1$ (mais un calcul analogue peut être mené dans le cas général).

Dans ce cas, la réponse impulsionelle du système caractérisé par $H(p)$ est définie par (voir paragraphe 5) :

$$
h(t)=\frac{1}{a^{n}} \sum_{k} p_{k} e^{t p_{k}}+\frac{\sin (n \pi)}{\pi} \int_{0}^{\infty} \frac{x^{n} e^{-t x}}{a^{2}-2 a x^{n} \cos (n \pi)+x^{2 n}} d x .
$$

La réponse $y(t)$ de ce système à une entrée $u(t)$ quelconque correspond au produit de convolution de $h(t)$ et de l'entrée $u(t)$, soit :

$$
y(t)=\int_{0}^{t} h(t-\tau) u(\tau) d \tau
$$

ou bien

$$
\begin{aligned}
y(t) & =\int_{0}^{t} \frac{1}{a^{n}} \sum_{k} p_{k} e^{(t-\tau) p_{k}} u(\tau) d \tau+ \\
& \int_{0}^{t} \frac{\sin (n \pi)}{\pi} \int_{0}^{\infty} \frac{x^{n} e^{-(t-\tau) x}}{a^{2}-2 a x^{n} \cos (n \pi)+x^{2 n}} u(\tau) d x d \tau
\end{aligned}
$$

ou encore

$$
\begin{aligned}
& y(t)=\int_{0}^{t} \frac{1}{a^{n}} \sum_{k} p_{k} e^{(t-\tau) p_{k}} u(\tau) d \tau+ \\
& \frac{\sin (n \pi)}{\pi} \int_{0}^{\infty} \frac{x^{n}}{a^{2}-2 a x^{n} \cos (n \pi)+x^{2 n}}\left[\int_{0}^{t} e^{-(t-\tau) x} u(\tau) d \tau\right] d x .
\end{aligned}
$$

Or la fonction

$$
w(t)=\left[\int_{0}^{t} e^{-(t-\tau) x} u(\tau) d \tau\right]
$$

correspond à l'expression de la réponse du système

$$
\dot{w}(t)=-x w(t)+u(t) \quad x>0
$$

Le système non entier $d$ 'origine admet donc la représentation d'état :

$$
\begin{aligned}
& {\left[\begin{array}{c}
\dot{w}_{1}(t) \\
\vdots \\
\dot{w}_{k}(t) \\
\dot{w}(t)
\end{array}\right]=\left[\begin{array}{cccc}
p_{1} & & & (0) \\
& \ddots & & \\
& & p_{k} & \\
(0) & & & -x
\end{array}\right]\left[\begin{array}{c}
w_{1}(t) \\
\vdots \\
w_{k}(t) \\
w(t)
\end{array}\right]+\left[\begin{array}{c}
1 \\
\vdots \\
1 \\
1
\end{array}\right] u(t)} \\
& y(t)=\left[\begin{array}{ccc}
\frac{p_{1}}{a_{n}} & \cdots & \frac{p_{k}}{a_{n}}
\end{array}\right]\left[\begin{array}{c}
w_{1}(t) \\
\vdots \\
w_{k}(t)
\end{array}\right]+ \\
& \frac{\sin (n \pi)}{\pi} \int_{0}^{\infty} \frac{x^{n} w(t, x)}{a^{2}-2 a x^{n} \cos (n \pi)+x^{2 n}} d x
\end{aligned}
$$


En appliquant le changement de variable $x=z^{2}$ soit $d x=2 z d z$, et en utilisant la transformée inverse de Fourrier spatial à l'équation (A.8) on montre alors que les équations d'état du système (A.1) peuvent être représentées par des équations faisant intervenir une équation de diffusion [18] :

$$
\begin{aligned}
& {\left[\begin{array}{c}
\dot{w}_{1}(t) \\
\vdots \\
\dot{w}_{k}(t)
\end{array}\right]=\left[\begin{array}{ccc}
p_{1} & & (0) \\
& \ddots & \\
(0) & & p_{k}
\end{array}\right]\left[\begin{array}{c}
w_{1}(t) \\
\vdots \\
w_{k}(t)
\end{array}\right]+\left[\begin{array}{c}
1 \\
\vdots \\
1
\end{array}\right] u(t)} \\
& \frac{\partial W(t, \xi)}{\partial t}=\frac{\partial W(t, \xi)}{\partial^{2} \xi}+u(t) \delta(\xi)
\end{aligned}
$$

Cette analyse renforce l'idée du lien étroit entre systèmes non entiers et phénomènes de diffusions.

\section{Bibliographie}

[1] K.S. Miller, B. Ross - An introduction to the fractional calculus and fractional differential equations - WileyInterscience Publication, 1993.

[2] S.G. Samko, A.A. Kilbas, O.I. Marichev - Fractional Integrals and Derivatives - Gordon and Breach Science Publishers, 1993.

[3] A. Oustaloup - La dérivation non entière, théorie, synthèse et applications - Editions Hermès, Paris, France, 1995.

[4] A. Oustaloup et B. Mathieu - La commande CRONE : du scalaire au multivariable - Editions Hermès, Paris, 1999.

[5] http://www.uniud.it/cism/corsi96/scaling/scope.html.

[6] P. Melchior, P. Lanusse, F. Dancla et O. Cois - CRONE Toolbox : une boîte à outils Matlab pour les systèmes fractionnaires - CETSIS-EEA'2001, pp. 241-244 Clermont-Ferrand, France, 29-30 Octobre 2001.

[7] P. Melchior, P. Lanusse, O. Cois, F. Dancla and A. Oustaloup - Crone Toolbox for Matlab: Fractional Systems Toolbox - Tutorial Workshop on "Fractional Calculus Applications in Automatic Control and Robotics" - 41st IEEE CDC'02 - Las Vegas, Nevada, USA, December 9-13, 2002.

[8] J. Sabatier, O. Cois et A. Oustaloup - Commande de systèmes fractionnaires par placement de pôles - $2^{\text {ème }}$ IEEE CIFA'2002 - Nantes, France, 6-8 Juillet 2002.

[9] J. Crank - The mathematics of diffusion - Oxford Univ. Press, London and New York, 1957.

[10] A. Oustaloup, Systèmes asservis linéaires d'ordre fractionnaire - Ed Masson, 1983.

[11] O. Cois, F. Levon, A. Oustaloup - Complex-fractional systems : modal decomposition and stability condition European Control Confernce ECC'2001, Porto, 2001.

[12] D. Matignon - Stability properties for generalized fractional differential systems - ESAIM: proc. Vol.5, Systèmes Différentiels Fractionnaires - Modèles, Méthodes et Applications, Paris, 1998.

[13] K. B. Oldham and J. Spanier - The fractional calculus: theory and applications of differentiation and integration to arbitrary order - Academic Press, New York and London, 1974.

[14] I. Podlubny - Fractional differential equations Mathematics in Sciences and Engineering $n^{\circ}$ 198, Academic Press, 1999.

[15] Y. Chen, K. L. Moore - Discretization schemes for fractional differentiators an integrators - IEEE Transaction on Circuits an Systems 1: Fundamental Theory and Applications, October, 2001.
[16] J. A. Tenreiro Machado - Discrete-time fractionalorder controllers - In proceedings of IEEE-INES'2000, 4th International Conference on Intelligent Engineering Systems, Portoroz, Slovenia, September 17-19, 2000.

[17] J. C. Trigeassou., T. Poinot, J. Lin, A. Oustaloup, F. Levron - Modeling and identification of a non integer order system - In: Proc ECC'99, European Control Conference, Karlsruhe, Germany, 1999.

[18] G. Montseny - Diffusive representation of pseudodifferential time-operators - ESAIM: proc. Vol.5, Systèmes Différentiels Fractionnaires - Modèles, Méthodes et Applications, Paris, 1998.

[19] L. Debnath - Recent applications of fractional calculus to Science and Engineering - IJMMS, Vol. 54, pp. 34133442, 2003.

[20] L. Le Lay - Identification fréquentielle et temporelle par modèle non entier - Thèse de Doctorat de l'Université Bordeaux 1, 20 Octobre 1998

[21] O. Cois et A. Oustaloup - Une synthèse sur les méthodes d'identification par modèle non entier - 3ème Conférence Internationale sur les Mathématiques Appliquées et les Sciences de l'Ingénieur, CIMASI'2000, Casablanca, Maroc, 23-25 Octobre 2000.

[22] O. Cois, A. Oustaloup, E. Battaglia and J.-L. Battaglia - Non integer model from modal decomposition for time domain system identification - 12th IFAC Symposiom on System Identification, SYSID'2000, Santa-Barbara, USA, June 21-23, 2000.

[23] M. Aoun, R. Malti, F. Levron and A. Oustaloup Orthonormal basis functions for modeling continuoustime fractional systems - 13th IFAC SYmposium on System Identification (SYSID'03), Rotterdam, The Netherlands, August 27-29, 2003.

[24] D. Matignon and B. d'Andréa-Novel - Some results on controllability and observability of finite-dimensional dractional differential systems - IMACS, IEEE-SMC Conference, pp. 952-956, Lille, France, 1996.

[25] R. Hotzel and M. Fliess - On linear systems with a fractional derivation: introductory theory and examples - Mathematics and computers in simulation, Vol. 45, pp 385-395, 1998.

[26] J. SABATIER, P. MELCHIOR et A. OUSTALOUP Réalisation d'un banc d'essais thermique pour l'étude des systèmes nonn entiers - CETSIS-EEA'2003, pp. 361364 - Toulouse, France, 13-14 Novembre 2003. 